\title{
Safety Ranges for Heart Rate Variability Parameters in Hyperbaric Environments
}

\author{
Carlos Sánchez ${ }^{1,2}$, Alberto Hernando ${ }^{1}$, Juan Bolea ${ }^{1,2}$, David Izquierdo ${ }^{1,3}$, María Teresa Lozano ${ }^{1,2}$, \\ María Dolores Peláez-Coca ${ }^{1,2}$ \\ ${ }^{1}$ Centro Universitario de la Defensa (CUD), Zaragoza, Spain \\ ${ }^{2}$ BSICoS Group, Aragón Inst of Engineering Research (I3A), IIS Aragón, Zaragoza, Spain \\ ${ }^{3}$ Photonic Technologies Group (GTF), Aragón Inst of Engineering Research (I3A), Zaragoza, Spain
}

\begin{abstract}
The Autonomic Nervous System (ANS) tries to maintain homeostasis in hyperbaric environments, but its activity may present large variability between subjects. The aim of this study is to establish safety ranges for ANS-related indices derived from the electrocardiographic signal (ECG) during diving and use them to identify subjects with abnormal ANS response and avoid potential diving accidents.

A database with ECG recordings from 28 subjects introduced into a hyperbaric chamber was used. During immersion, five stages were studied at 1, 3 and 5 atm during descent and ascent. Indices of heart rate variability, extracted from ECG, reflecting the sympathetic and parasympathetic ANS response, were calculated and regularised with respect to their values at the initial stage at $1 \mathrm{~atm}$. In particular, four time-related parameters extracted from the RR series and four frequency parameters based on the powers of the low and high frequency bands were used.

High inter-subject variability in the ANS response was observed in all stages. The eight parameters were analysed for each stage and, as a result, some subjects presented highly uncommon responses with higher chances of suffering a diving accident, reflected in many parameters out of the interquartile range. This allows establishing safety ranges for ANS-related parameters that can help in the identification of subjects with potential health risk.
\end{abstract}

\section{Introduction}

Some common activities that human beings have learnt to do may represent a physiological challenge for the body to maintain homeostasis while adapting to the surrounding conditions. One of them is diving in deep waters, which can be risky if not performed in a controlled manner. Water density is about 800 times higher than air density, so a descent of $10 \mathrm{~m}$ in water supposes an increase in barometric pressure of $1 \mathrm{~atm}$.
The changes in partial gas concentrations in human respiratory system that occur at high pressures may eventually cause decompression sickness, with severe associated health problems if those pressure variations are not performed gradually. Increased pressure entails changes in cardiac function: increase in the systolic volume and heart rate (HR) reduction in order to maintain an adequate cardiac output to minimize the impact on the body [1]. This adaptation is possible thanks to the response of the Autonomic Nervous System (ANS). The assessment of the response of the ANS to large changes in pressure is challenging and may present large variability between subjects. Studies aiming at monitoring and controlling this response under these extreme conditions, as well as identifying potential risks for divers' health are of great interest, for example in the training of military personnel.

In this study, the heart rate variability signal (HRV) is extracted from the electrocardiographic signal (ECG), where ANS response, reflected in the activity of its sympathetic and parasympathetic branches, is evaluated by HRV derived indices [2].

The goals of this study are mainly two: to characterize ranges of indices for normal ANS response to changes in barometric pressure using a hyperbaric chamber and to identify subjects with abnormal ANS responses in these environments.

\section{Materials and methods}

\subsection{Database}

The database used in this study consisted of 28 volunteers ( 25 males and 3 females), with a mean age of $28.5 \pm$ 6.2 years. All the volunteers gave written consent validated by the Ethics Committee.

ECG and respiration signals of all subjects were recorded inside a hyperbaric chamber of the Hospital General de la Defensa en Zaragoza. The device used for ECG 
recording was Nautilus, developed by the University of Kaunas, Lithuania [3]. This device allowed us to record the ECG signal with three non-orthogonal leads at a sampling frequency of $2000 \mathrm{~Hz}$.

The protocol inside the hyperbaric chamber had a duration of about 2 hours of immersion up to a maximum of $5 \mathrm{~atm}$ in pressure with time intervals in agreement with the decompression table recommendations (see www.naui.org/resources/). During immersion, five stops lasting five minutes each were performed at 1, 3 and 5 atm during descent (D) and ascent (A), and subsequently analysed: from 1D (basal state) to 3D, 5 (maximum depth), 3A and back to $1 \mathrm{~A}$.

\subsection{Heart rate variability analysis}

Delineation of the recorded ECG signal to detect the position of the heart beats was performed using an algorithm based on the wavelet transform [4]. Ectopic beats, missed beats and false detections were corrected [5]. Then, the series of R-wave intervals were used to compute the instantaneous HR signal at a sampling rate of $4 \mathrm{~Hz}$ using the integral pulse frequency modulation model [6]. Low frequency modulation of HR, i.e. mean heart rate, was subtracted by low-pass filtering at $0.03 \mathrm{~Hz}$ in order to obtain HRV signal.

Eight ECG-derived indices from the HRV signal, which reflect the sympathetic and parasympathetic ANS response, were calculated and regularised with respect to their values at the reference stage 1D.

Some parts of the ECG recordings were discarded when superposition of respiratory rate on low frequency band appeared or poor quality recordings were identified. This reduced the number of valid subjects for the analysis to 15 in stages $3 \mathrm{D}$ and $3 \mathrm{~A}$, and 13 in stages 5 and $1 \mathrm{~A}$.

\subsection{Time-domain $\mathrm{HRV}$ indices}

Four time-domain parameters were computed from the $\mathrm{R}$-wave interval series, and then their averaged values in the last four minutes for each immersion stage were obtained:

- $N N(s)$ : median of the Normal-to-Normal intervals. - $\operatorname{IQR}(s)$ : interquartile range of $N N$ intervals as a measure of statistical dispersion.

- $R M S S D(s)$ : root mean square of the successive differences between adjacent $N N$ intervals.

- $p N N 50(\%)$ : number of pairs of successive $N N$ intervals that differ by more than $50 \mathrm{~ms}$, divided by the total number of $N N$ intervals.

\subsection{Frequency-domain HRV indices}

Four classic frequency-domain parameters were calculated based on the power spectral distribution analysis of the HRV signal. This analysis was performed using the smoothed pseudo Wigner-Ville distribution, since it provides a trade-off between time-frequency resolution, as well as low variance power estimations. To reduce the impact of interfering terms in time and frequency, smoothing Hamming windows with lengths of 203 samples (in time) and 513 samples (in frequency) were used, respectively [7]. The four indices were based on the powers of the classical bands of low frequency (LF, $0.04-0.15 \mathrm{~Hz}$ ) and high frequency (HF, $0.15-0.4 \mathrm{~Hz}$ ) of the HRV spectrum in the last four minutes of each stage:

- $P_{L F}($ a.u.): mean of the power in the LF band.

- $P_{H F}($ a.u.): mean of the power in the HF band.

- $P_{L F_{n}}$ (n.u.): mean of the power in the LF band over the sum of the powers of both LF and HF bands. $P_{L F_{n}}=$ $P_{L F} /\left(P_{L F}+P_{H F}\right)$.

- $R_{L F / H F}$ (n.u.): mean of the ratio between the power in the LF band and the power in the HF band. $R_{L F / H F}=$ $P_{L F} / P_{H F}$.

\subsection{Parameter regularisation}

The average of the eight index values of the last four minutes of each stage of immersion were calculated. Then, they were regularised with respect to the values of the reference stage $1 \mathrm{D}$ as follows, so that all of them are in the $[0,1]$ range:

$$
R\left(Y_{S}\right)=\frac{Y_{S}}{Y_{S}+Y_{1 D}},
$$

where $Y$ is the parameter to regularise and $S$ is the stage $(3 \mathrm{D}, 5,3 \mathrm{~A}$ or $1 \mathrm{~A})$.

\subsection{Statistical analysis}

The regularised parameters were analysed to evaluate whether the subjects presented abnormal ANS responses to changes in pressure during the different stages of immersion. This analysis was conducted separately for each subject of the database in two steps:

1) Leave-one-out: interquartile (25-75 percentile) and 595 percentile ranges were computed leaving the analysed subject out.

2) The number of regularised indices from the analysed subject that were out of the ranges obtained in step 1) were calculated.

\section{Results}

Inter-subject variability in the four stages is notable in all the parameters except for NN and RMSSD, whose reg- 

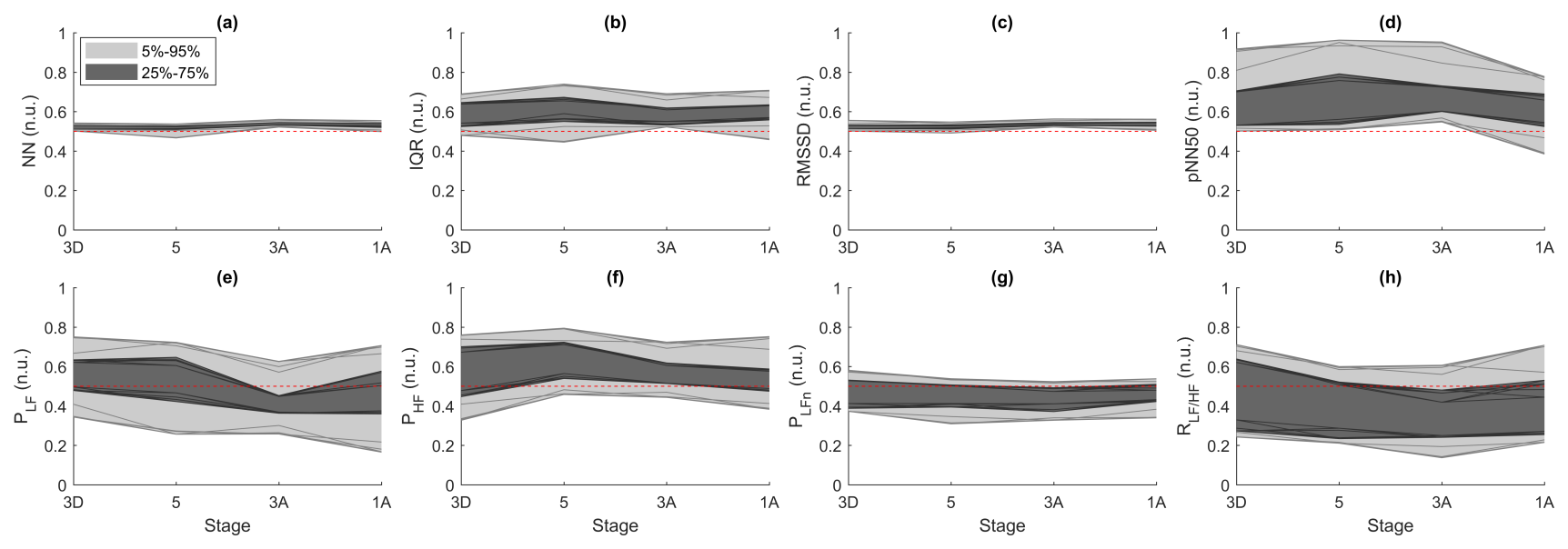

Figure 1. Interquartile (25-75; dark grey) and 5-95 percentile (light grey) ranges of the eight regularised parameters in the four stages of immersion. Solid lines represent the variations in the limits of the ranges when each subject is left out. Dashed lines are the reference values, i.e. no changes with respect to stage 1D.

ularised values are slightly larger than 0.5 for all subjects and stages (Figure 1, panels (a) and (c)). Therefore, the ANS response of subjects showing relatively small differences in these parameters with respect to the rest of subjects would make them be classified as abnormal. In contrast, pNN50 is increased in nearly all subjects and stages with respect to the reference stage (values above 0.5), and with large variability between subjects in both interquartile range $(\approx 0.15-0.2$ n.u. $)$ and $5-95$ percentile range $(\approx 0.4$ n.u.) (Figure 1, panel (d)).

Regarding the powers in LF and HF bands, interquartile and 5-95 percentile ranges are relatively wide with a decreasing trend in $P_{L F}$ as the immersion progresses (width of $\approx 0.15-0.2$ n.u. in interquartile range, and $\approx 0.3-0.35$ n.u. in the 5-95 percentile range; Figure 1, panel (e)). On the other hand, $P_{H F}$ exhibits a bi-phasic behaviour with larger values at higher pressures, as shown in previous studies [8,9], interquartile range decreasing in width (from $\approx 0.25$ n.u. at stage $3 \mathrm{D}$ to $\approx 0.1$ n.u. at stage $1 \mathrm{~A}$ ), and 5-95 percentile range maintaining a width of $\approx 0.35$ - 0.4 n.u. in all stages (Figure 1, panel (f)). It is worth noting that the ratios of powers behave differently: the ranges of $P_{L F_{n}}$ do not show relevant changes between stages with widths of $\approx 0.15$ n.u. and $\approx 0.2$ n.u. in interquartile range and 5-95 percentile range, respectively (Figure 1, panel (g)), whereas the ranges of $R_{L F / H F}$ are notably wider implying significant intersubject variability in all stages (widths of $\approx 0.4$ n.u. in interquartile range, and $\approx 0.5$ n.u. in 5-95 percentile range; Figure 1, panel (h)).

Figure 2 shows the classification of each specific subject according to the number of the eight regularised parameters that are either out of the interquartile range (top panel) or out of the 5-95 percentile range (bottom panel). Due to the removal of some parts of the ECG in some sub- jects, not all of them are present in the analysis of the four stages of immersion (15 valid for stages $3 \mathrm{D}$ and $3 \mathrm{~A} ; 13$ valid for stages 5 and $1 \mathrm{~A}$ ). The most interesting findings of this analysis are:

- No subjects have all parameters within the interquartile range in the four stages, meaning that a subject showing a common ANS response in a specific stage (i.e. low number of parameters out of interquartile range) does not guarantee to be the case in the rest of immersion (see subjects \#4, \#27 or \#28).

- Some subjects present almost all their parameters within the 5-95 percentile range in the four stages, but with a relevant number of them out of the interquartile range in some stages (see subjects \#6 and \#9).

- Some subjects have many parameters out of the interquartile range in the four stages of immersion (see subject \#17).

\subsection{Limitations}

The complete database is not very large due to the implicit difficulties that entail recordings in hyperbaric chamber. Therefore, removal of signal segments with low respiratory frequency or low quality, and thus removal of the subject from the analysis of a particular stage, implies a relevant limitation in the analysis performed in this study. Some subjects breath slowly at the reference stage, but not during the immersion. Separation of the effects on HRV due to respiration and those due to pressure changes is challenging and needs to be further explored.

\section{Conclusion}

The analysis performed in this study is a first approach to establishing safety ranges for ANS-related parameters 
\# subject with 'Np' params out $\mathbf{2 5 - 7 5 \%}$

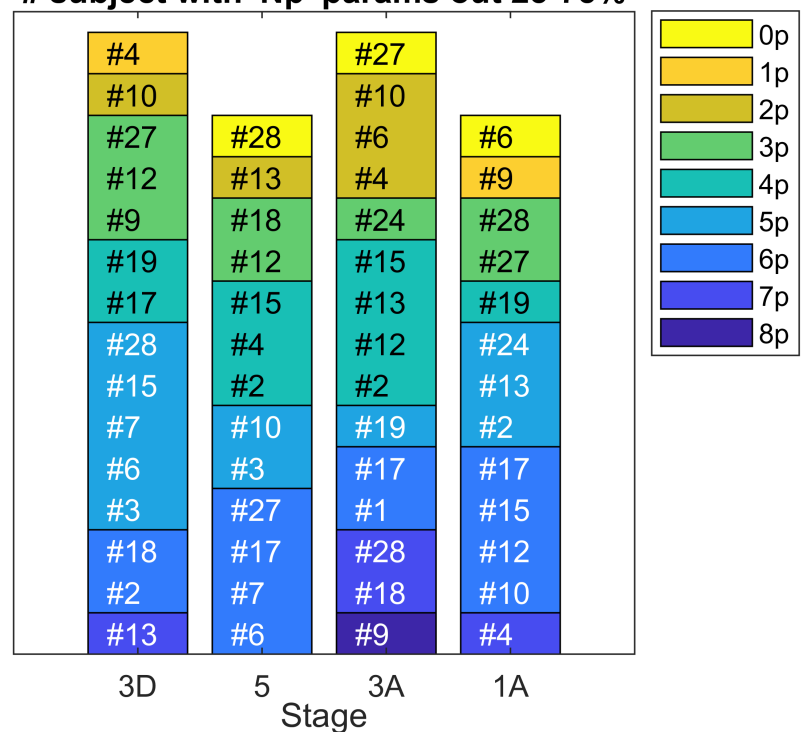

\# subject with 'Np' params out 5-95\%

\begin{tabular}{|c|c|c|c|c|}
\hline \#19 & & \#28 & & $0 p$ \\
\hline \#17 & & \#27 & & $1 p$ \\
\hline \#15 & \#28 & \#12 & \#27 & $3 n$ \\
\hline \#10 & \#18 & \#10 & \#12 & $4 p$ \\
\hline \#9 & \#12 & \#9 & \#9 & $5 p$ \\
\hline \#4 & \#4 & \#6 & \#6 & $6 p$ \\
\hline \#2 & \#3 & \#19 & \#28 & $7 p$ \\
\hline \#6 & \#17 & \#15 & \#24 & $8 p$ \\
\hline \#28 & \#13 & \#13 & $\# 17$ & \\
\hline \#27 & \#6 & \#4 & \#13 & \\
\hline \#12 & \#10 & \#2 & \#10 & \\
\hline \#7 & \#2 & \#1 & \#19 & \\
\hline \#18 & \#7 & \#24 & \#2 & \\
\hline \#3 & \#27 & $\# 17$ & $\# 15$ & \\
\hline \#13 & \#15 & \#18 & \#4 & \\
\hline $3 D$ & 5 & $3 A$ & $1 \mathrm{~A}$ & \\
\hline
\end{tabular}

Figure 2. Specific subjects (\#) with 'Np' parameters (color scale, from ' $0 \mathrm{p}$ ' to ' $8 \mathrm{p}$ ') out of the interquartile range (top panel), and out of the 5-95 percentile range (bottom panel) in each stage.

in a controlled simulated immersion in a hyperbaric chamber. This can be helpful for the identification of subjects with potential risk for their health in real diving or other hyperbaric activities with more uncontrolled factors, thus discouraging their execution. Extending the database and including more parameters could be of high interest to increase robustness of the results.

\section{Acknowledgements}

This work has been partially financed by Ministerio de Economía, Industria y Competitividad, FEDER and Centro Universitario de la Defensa through the projects CUD2019-10, UZ-CUD2019-TEC-01, and PGC2018095936-B-I00. The authors would like to thank Hospital General de la Defensa en Zaragoza, Regimiento de Pontoneros y Especialidades de Ingenieros $\mathrm{n}^{\mathrm{o}} 12$ for their valuable collaboration, and the consolidated research group BSICoS for the algorithms facilitated.

\section{References}

[1] Widmaier EP. Vander's human physiology: the mechanisms of body function. 11th edition. Boston: McGraw-Hill Higher Education.

[2] Heart rate variability: standards of measurement, physiological interpretation and clinical use. Task Force of the European Society of Cardiology and the North American Society of Pacing and Electrophysiology. Circulation March 1996; 93(5):1043-1065.

[3] Sokas D, et al. Diver physiology monitor and its graphical user interface. In Proceedings of International Scientific - Practical Conference, Virtual Instruments in Biomedicine. 2016; 5-9.

[4] Martinez J, et al. A wavelet-based ECG delineator: evaluation on standard databases. IEEE Transactions on Biomedical Engineering April 2004;51(4):570-581.

[5] Mateo J, et al. Analysis of heart rate variability in the presence of ectopic beats using the heart timing signal. IEEE Transactions on Biomedical Engineering March 2003; 50(3):334-343.

[6] Bailón R, et al. The integral pulse frequency modulation model with time-varying threshold: application to heart rate variability analysis during exercise stress testing. IEEE Transactions on Biomedical Engineering March 2011; 58(3):642-652.

[7] Bailón R, et al. Influence of running stride frequency in heart rate variability analysis during treadmill exercise testing. IEEE Transactions on Biomedical Engineering July 2013;60(7):1796-1805.

[8] Hernando A, et al. Autonomic nervous system measurement in hyperbaric environments using ECG and PPG signals. IEEE Journal of Biomedical and Health Informatics 2018;1-1.

[9] Sánchez C, et al. Autonomic nervous system non-stationary response to controlled changes in barometric pressure. In 2017 Computing in Cardiology (CinC). September 2017; 14.

Address for correspondence:

Carlos Sánchez Tapia

Centro Universitario de la Defensa (CUD)

Academia General Militar (AGM)

cstapia@unizar.es 